\title{
Forecasting RMB Exchange Rate Based on a Nonlinear Combination Model of ARFIMA, SVM, and BPNN
}

\author{
Chi Xie, ${ }^{1,2}$ Zhou Mao, ${ }^{1}$ and Gang-Jin Wang ${ }^{1,2}$ \\ ${ }^{1}$ College of Business Administration, Hunan University, Changsha 410082, China \\ ${ }^{2}$ Center of Finance and Investment Management, Hunan University, Changsha 410082, China
}

Correspondence should be addressed to Gang-Jin Wang; wanggangjin@hnu.edu.cn

Received 7 May 2014; Revised 25 July 2014; Accepted 25 July 2014

Academic Editor: Erol Egrioglu

Copyright (C) 2015 Chi Xie et al. This is an open access article distributed under the Creative Commons Attribution License, which permits unrestricted use, distribution, and reproduction in any medium, provided the original work is properly cited.

\begin{abstract}
There are various models to predict financial time series like the RMB exchange rate. In this paper, considering the complex characteristics of RMB exchange rate, we build a nonlinear combination model of the autoregressive fractionally integrated moving average (ARFIMA) model, the support vector machine (SVM) model, and the back-propagation neural network (BPNN) model to forecast the RMB exchange rate. The basic idea of the nonlinear combination model (NCM) is to make the prediction more effective by combining different models' advantages, and the weight of the combination model is determined by a nonlinear weighted mechanism. The RMB exchange rate against US dollar (RMB/USD) and the RMB exchange rate against Euro (RMB/EUR) are used as the empirical examples to evaluate the performance of NCM. The results show that the prediction performance of the nonlinear combination model is better than the single models and the linear combination models, and the nonlinear combination model is suitable for the prediction of the special time series, such as the RMB exchange rate.
\end{abstract}

\section{Introduction}

With the persistent development of economic globalization, business contacts among nations get increasingly frequent. As international trade and financial activities are closely related to exchange rate, the status of exchange rate is very prominent. Ding and Ying [1] analyzed the relationship between exchange rate and export trade. The results indicated that changes of exchange rate not only affect the national economic balance but also have an impact on the level of foreign trade, which further influences the economic development of the relevant country. Currently, because China's economy occupies an important position in the global economy, the Renminbi (RMB) exchange rate has a pivotal position in the stability and development of the world economy. For the past few years, with the propulsion of opening and reforming in China, the value of its currency has determined by the market step by step. Under the floating exchange rate system, the exchange rate volatility shows some characteristics: frequent, intense, and sudden. The RMB exchange rate prediction plays a very important role in policy setting, foreign trade, and investment activity.

Traditionally, the exchange rate prediction methods can be classified into two types: fundamental analysis and techni$\mathrm{cal}$ analysis. The assumption of fundamental analysis is that the changes of the exchange rate are caused by the changes of the purchasing power ratio of the two countries. For instance, Grossmann and Simpson [2] used the improved purchasing power parity (PPP) model to forecast the exchange rate. In recent years, due to the increasingly complicated international economic and financial environment, the basic economic factors which affect exchange rates are changing all the time. $\mathrm{Hu}$ [3] suggested that the terms of trade, the national openness, and the level of domestic technology have an impact on the RMB exchange rate. It is difficult to predict the RMB exchange rate by the fundamental analysis. However, as a time series, no matter how exchange rate system changes, there is a stable internal structure in the exchange rate. The assumption of technical analysis is that a nonlinear correlation structure exists in time series. Therefore, using 
implicit information of time series to make trend analysis is easy to implement, and the exchange rate time series prediction model has a great development. Dai and Xiao [4], $\mathrm{Xu}$ and $\mathrm{Li}$ [5], and $\mathrm{Ma}$ and $\mathrm{Xu}$ [6] made a prediction of the exchange rate against US dollar by the autoregressive integrated moving average (ARIMA) model.

Sun and $\mathrm{Wu}$ [7], Rong [8], and Xie et al. [9] discovered the nonlinear correlation structure of the RMB exchange rate by the R/S method. Considering the nonlinearity of the $\mathrm{RMB}$ exchange rate time series, many scholars studied the nonlinear forecasting methods, and the nonlinear methods become popular gradually. For example, Yang and $\mathrm{Wu}$ [10] showed that the support vector machine (SVM) model has the ability of prediction. Kadilar et al. [11], Aladag et al. [12], and Aladag and Marinescu [13] used the neural networks $(\mathrm{NN})$ to forecast exchange rate series. Katijani et al. [14] employed the feed forward neural networks (FNN) to forecast nonlinear time series. Panda and Narasimhan [15] compared the NN model with the autoregressive (AR) model and the random walk (RW) model and proved that nonlinear models are more effective than linear models in forecasting exchange rate time series.

Many studies on predictions indicate that single models cannot fit and analyze the complex characteristics of exchange rate time series effectively [16]. Furthermore, different models have similarities in data mining and analysis; that is to say, there is a correlation among models. In order to improve the forecasting ability, a large number of scholars mixed different forecast models [17]. For instance, Alpaslan et al. [18], Alpaslan and Cagcag [19], and Cagcag Yolcu [20] came up with a hybrid fuzzy approach to analyze time series. Then, Luna et al. [21], Rojas et al. [22], Wong et al. [23], and Zhao and Yang [24] used the fuzzy clustering and artificial neural network (ANN) to solve the problem of time series forecasting. Aladag et al. [25] proposed a new hybrid approach by combining Elman's recurrent neural networks (ERNN) and ARIMA models to forecast the nonlinear times series like the Canadian Lynx data. Egrioglu et al. [26] constructed a hybrid model of seasonal ARIMA, autoregressive conditional heteroscedasticity (ARCH), and ANN to forecast nonlinear time series. By considering that some time series have the long memory characteristic, Aladag et al. [27] combined the autoregressive fractionally integrated moving average (ARFIMA) models and FNN to forecast the tourism data of Turkey. Valenzuela et al. [28] and Khashei and Bijari [29] proved that the prediction of ARIMA-ANN hybrid model is more accurate than single models.

In this paper, we propose a nonlinear combination model to capture the complex characteristics of RMB exchange rate time series. In general, combination models use different models to forecast linear and nonlinear portions, respectively, and combine their results by linear or nonlinear methods. Because the fitting processes of most of the models are similar, their forecast results have some correlations. So, in view of that there are linear and nonlinear relationships among different models, we use the nonlinear function to combine different models. Our approach, called the nonlinear combination model (NCM), combines the ARFIMA model, the back-propagation neural network (BPNN) model, and the SVM model. The ARFIMA mainly simulates the linear relationship and captures the long memory feature of foreign exchange rate, while the SVM and BPNN seize the nonlinear relationship, and the weights of these models are determined by the nonlinear weighted mechanism. Although many research groups have forecasted exchange rate by various models, the focus of our work is on considering the relationship between different models and combining their forecasting abilities. The nonlinear combination model can reflect the characteristics of the exchange rate and make the forecasting more accurate.

To evaluate the performance of the proposed approach, we use RMB/USD and RMB/EUR as the empirical examples. The results show that the NCM outperforms all component models used alone and the equal weights linear (EWL) model. In addition, our model reveals more robust features with regard to the possible structure changes in the data.

The rest of the paper is organized as follows. Section 2 presents the component models of ARFIMA, SVM, and BPNN briefly. The construction of the nonlinear combination model is described in Section 3. Section 4 presents the experimental results based on the real $\mathrm{RMB}$ exchange rate time series. Finally, Section 5 is a summary of implications and conclusions.

\section{Individual Forecasting Models Used in the Nonlinear Combination Model}

2.1. Autoregressive Fractionally Integrated Moving Average (ARFIMA) Model. The autoregressive fractionally integrated moving average (ARFIMA) model is a linear model introduced by Granger and Joyeux [30] and is widely used to fit and forecast time series. The $\operatorname{ARFIMA}(p, d, q)$ model is represented as follows [30, 31]:

$$
\Psi(B)(1-B)^{d} X_{t}=\Theta(B) e_{t}
$$

where $B$ is the back-shift operator such that $B X_{t}=X_{t-1} ; e_{t}$ is a white noise process; $-0.5<d<0.5 ; \Psi(B)=1-\Psi_{1} B-$ $\cdots-\Psi_{p} B^{p} ; \Theta(B)=1-\Theta_{1} B-\cdots-\Theta_{q} B^{q} ; p$ is the number of the autoregressive terms; $d$ is the number of the fractional differences; and $q$ is the number of the lagged forecast errors in the prediction equation.

We obtain the following equation by the binomial expansion:

$$
\begin{aligned}
(1-B)^{d}= & \sum \frac{d !}{k !(d-k) !}(-1)^{k} B^{k} \\
= & 1-d B+\frac{d(d-1) B^{2}}{2 !} \\
& -\frac{d(d-1)(d-2) B^{3}}{3 !}+\cdots,
\end{aligned}
$$


where

$$
\begin{aligned}
\sum & \frac{d !}{k !(d-k) !}(-1)^{k} B^{k} \\
& =\frac{\Gamma(d+1)}{\Gamma(k-1) \Gamma(d-k+1)} \\
& = \begin{cases}\frac{d(d-1)(d-2) \cdots(d-k+1)}{k !}, & k>0 \\
1, & k=0,\end{cases}
\end{aligned}
$$

where $\Gamma(x)$ is the Gamma function.

When $-0.5<d<0$, ARFIMA $(p, d, q)$ is a short memory process; when $0<d<0.5$, ARFIMA $(p, d, q)$ is a long memory process; when $0.5<d<1$, ARFIMA $(p, d, q)$ is a mean reverting process. Some scholars proved that $d$ has a certain relationship with the Hurst value [32] as

$$
\text { Hurst }=0.5+d \text {. }
$$

Especially, when $d$ is an integer, the ARFIMA model reduces to ARIMA model. As proposed by Box and Jenkins [33], the ARIMA $(p, d, q)$ model is defined as follows:

$$
\begin{aligned}
w_{t}= & \Delta^{d} y_{t} \\
w_{t}= & \phi_{1} w_{t-1}+\cdots+\phi_{p} w_{t-p} \\
& +\varepsilon_{t}-\theta_{1} \varepsilon_{t-1}-\cdots-\theta_{q} \varepsilon_{t-q}, \\
& t \in T,
\end{aligned}
$$

where $y_{t}$ is the actual value; $w_{t}$ is the stationary time series by a $d$ th integer difference; $\phi_{i}(i=1,2, \ldots, p)$ and $\theta_{j}(j=$ $1,2, \ldots, q)$ are the autoregressive coefficients and residual coefficients, respectively; and $\varepsilon_{t}$ is the white noise.

2.2. Support Vector Machine (SVM) Model. The support vector machine (SVM) model is proposed by Vapnik [34]. It is a learning model without parameters. The principal of SVM model is to construct an optimal separating hyperplane to make correct classification of true output value. The SVM model tries to model the learning method of training data as a function as follows: $f(x)=w \tau(x)+b$, where $w$ is the weight vector, $\tau$ is the nonlinear mapping to a high dimensional feature space, and $b$ is bias. The optimal hyperplane function through minimizing the risk by loss function is as follows:

$$
y(x)=\sum_{i=1}^{N_{s}}\left(\alpha_{i}-\alpha_{i}^{*}\right) K\left(x, x_{i}\right)+b_{\mathrm{opt}}
$$

where $\alpha_{i}$ and $\alpha_{i}^{*}\left(i=1,2, \ldots, N_{s}\right)$ are the Lagrange multipliers; $N_{s}$ is the number of support vectors; $K$ is the kernel function; and $b_{\text {opt }}$ is the ideal bias. Model parameters are chosen by cross validation techniques. The usual kernel function is the radial basis function as follows:

$$
K\left(x, x_{i}\right)=\exp \left(\frac{-\left\|x-x_{i}\right\|^{2}}{2 \sigma^{2}}\right) .
$$

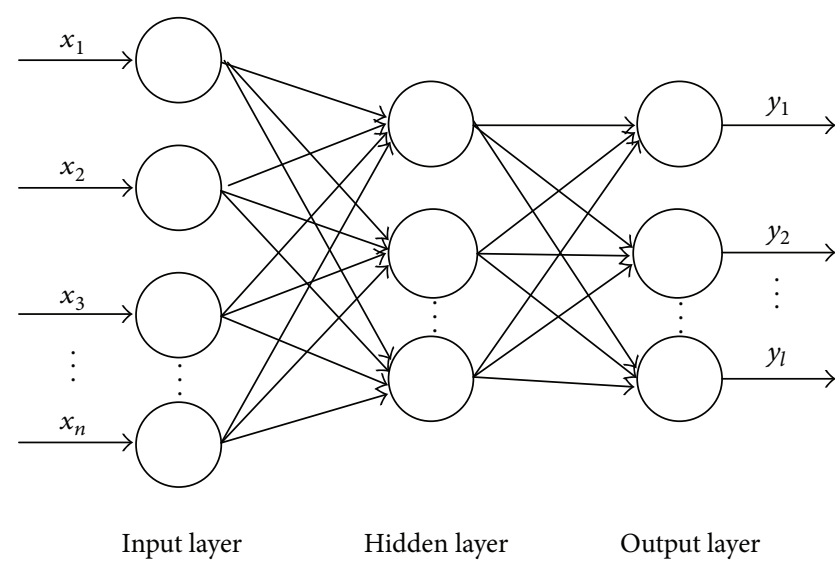

FIGURE 1: The structure of BPNN.

2.3. Back-Propagation Neural Network (BPNN) Model. Backpropagation neural network (BPNN) model is a feedback network introduced by Rumelhart and McClelland [35], and its structure is similar to the neurons of the brain which transmit signals through the synapse. BPNN has an internal structure composed of input layer, hidden layer, and output layer. Each layer contains one or more neurons. Although there is no mutual connection between nodes of the same layer, neurons of adjacent layers can connect through the adjustable weights, and there is no feedback between the neurons. The BPNN model consists of $n$ input layer neurons, $m$ hidden layer neurons, and $l$ output layer neurons. The network structure of BPNN is shown in Figure 1.

When BPNN receives the input signals, it transmits the signals to the hidden layer like the operation process of the human neurons. Then, the BPNN model transmits the signals from the hidden layer to the output layer, and each neuron in the output layer gets the signals of the input layer. In the BPNN model, we train the learning process before predicting. First of all, we set the initial connection weights. Then, in order to reduce the errors between the actual output and the target output, we correct the weights gradually from the output layer to the input layer.

The calculation process of the output data in hidden layer neurons is as follows:

$$
\begin{aligned}
& \text { net }_{i}=\sum_{j=1}^{n} w_{j i} x_{j}, \quad j=1,2, \ldots, n, \\
& z_{i}=f_{H}\left(\text { net }_{i}\right), \quad i=1,2, \ldots, m,
\end{aligned}
$$

where $x_{j}(j=1,2, \ldots, n)$ are the input layer data; $w_{j i}$ is the weight from the input layer neuron $j$ to the hidden layer neuron $i$; net $_{i}(i=1,2, \ldots, m)$ are the values from the input layer to the hidden layer neuron $i ; f_{H}$ is the node function; and $z_{i}(i=1,2, \ldots, m)$ are the operation values of neuron $i$. $f_{H}$ is often used as sigmoid function as follows:

$$
f_{H}(x)=\frac{1}{1+\exp (-x)} .
$$


The calculation process of the output data in output layer neurons is as follows:

$$
y_{t}=f_{T}\left(\sum_{i=1}^{m} w_{i t} z_{i}\right), \quad t=1,2, \ldots, l,
$$

where $y_{t}(t=1,2, \ldots, l)$ are the output layer data and $w_{i t}$ is the weight from the hidden layer neuron $i$ to the output layer neuron $t ; f_{T}$ is the output layer conduction function, which usually is expressed as a linear function.

\section{Nonlinear Combination Model}

3.1. Construction of the Nonlinear Combination Model. How to combine different models is an extensively studied problem in the academic research. Hibon and Evgeniou [36] demonstrated that the prediction ability of the hybrid model was better than a single model. So, Hassan et al. [37] combined the hidden Markov model, ANN, and genetic algorithm to construct a hybrid model for predicting stock index time series. Aladag et al. [38] combined different models by using artificial neural networks to forecast the index of Istanbul stock exchange (IMKB) time series.

The principal of the nonlinear combination forecasting model is to make a certain nonlinear combination of various results from different forecast models. In the forecasting process, we combine the different models by a nonlinear function. The prediction results of nonlinear combination model are as follows:

$$
\begin{aligned}
\widehat{v}_{t}^{(i)}= & \frac{\left(\widehat{y}_{t}^{(i)}-\mu^{(i)}\right)}{\left(\sigma^{(i)}\right)^{2}}, \\
\widehat{y}_{t}^{(c)}= & w_{0}+w_{1} \widehat{y}_{t}^{(1)}+w_{2} \widehat{y}_{t}^{(2)}+\cdots+w_{n} \widehat{y}_{t}^{(n)} \\
& \quad+\theta_{1} \widehat{v}_{t}^{(1)} \widehat{v}_{t}^{(2)}+\theta_{2} \widehat{v}_{t}^{(2)} \widehat{v}_{t}^{(3)}+\cdots+\theta_{n} \widehat{v}_{t}^{(n)} \widehat{v}_{t}^{(1)}, \\
\widehat{Y}^{(c)}= & {\left[\widehat{y}_{1}^{(c)}, \widehat{y}_{2}^{(c)}, \ldots, \widehat{y}_{N}^{(c)}\right]^{T}, }
\end{aligned}
$$

where $\widehat{y}_{t}^{(i)}(i=1,2, \ldots, n)$ are the prediction results of model $i ; \mu^{(i)}(i=1,2, \ldots, n)$ and $\sigma^{(i)}(i=1,2, \ldots, n)$ are the means and standard deviations of the sequence $\widehat{y}_{t}^{(i)}$ of model $i$, respectively; $w_{i}(i=0,1, \ldots, n)$ and $\theta_{j}(j=1,2, \ldots, n)$ are the weights of the nonlinear combination model; and $\widehat{Y}^{(c)}$ is the prediction results of the nonlinear combination model.

3.2. Determination of the Combination Weights. Determining the weights for combination model is a key process in improving the prediction ability. There are various methods to combine different models, and the simplest one is the linear equal weight method. However, the linear equal weight method fails to notice that there are nonlinear relationships among different models. Thus, we propose another weighted mechanism to determine the parameters of the nonlinear function.

Our proposed weighted mechanism determines the corresponding parameters by minimizing the square sum of the errors (SSE) between the actual and estimated values.
But the SSE of testing set is unknown in advance; so, we divide time series into training subsets and validation subsets. The size of the validation set is equal to the size of the testing set. The individual forecasting models are then trained on the training set and the nonlinear function parameters are calculated by minimizing the SSE of validation set as

$$
\mathrm{SSE}=\sum_{t=1}^{N}\left(y_{t}-\widehat{y}_{t}^{(c)}\right)^{2}
$$

To minimize SSE, the following conditions must be satisfied:

$$
\frac{\partial \mathrm{SSE}}{\partial w_{i}}=0, \quad \frac{\partial \mathrm{SSE}}{\partial \theta_{j}}=0,
$$

where $i=0,1, \ldots, n$ and $j=1,2, \ldots, n$.

We simplify it and get the following equations:

$$
\begin{aligned}
& V W+Z \theta=b, \quad Z^{T} W+U \theta=d, \\
& W=\left[w_{0}, w_{1}, w_{2}, w_{3}\right]^{T}, \quad \theta=\left[\theta_{1}, \theta_{2}, \theta_{3}\right], \\
& V=F^{T} F, \quad Z=F^{T} G, \quad U=G^{T} G, \\
& b=F^{T} Y, \quad d=G^{T} Y, \\
& F=\left[\begin{array}{cccc}
1 & \widehat{y}_{1}^{(1)} & \widehat{y}_{1}^{(2)} & \widehat{y}_{1}^{(3)} \\
\vdots & \vdots & \vdots \\
1 & \widehat{y}_{N}^{(1)} & \widehat{y}_{N}^{(2)} & \widehat{y}_{N}^{(3)}
\end{array}\right]_{N \times 4}, \\
& G=\left[\begin{array}{ccc}
\widehat{v}_{1}^{(1)} \widehat{v}_{1}^{(2)} & \widehat{v}_{1}^{(2)} \widehat{v}_{1}^{(3)} & \widehat{v}_{1}^{(3)} \widehat{v}_{1}^{(1)} \\
\vdots & \vdots & \vdots \\
\widehat{v}_{N}^{(1)} \widehat{v}_{N}^{(2)} & \widehat{v}_{N}^{(2)} \widehat{v}_{N}^{(3)} & \widehat{v}_{N}^{(3)} \widehat{v}_{N}^{(1)}
\end{array}\right]_{N \times 3} .
\end{aligned}
$$

Then, we calculate the parameters according to the following equations:

$$
\begin{aligned}
& \theta_{\mathrm{opt}}=\left(U-Z^{T} V^{-1} Z\right)^{-1}\left(d-Z^{T} V^{-1} b\right) \\
& w_{\mathrm{opt}}=V^{-1}\left(b-Z \theta_{\mathrm{opt}}\right) .
\end{aligned}
$$

3.3. Evaluation Criteria. In order to evaluate the forecasting performance of the nonlinear combination model, we use the mean absolute error (MAE), the root mean square error (RMSE), and the mean absolute percentage error (MAPE). These are defined as follows:

$$
\begin{aligned}
\text { MAE } & =\frac{1}{N} \sum_{t=1}^{N}\left|y_{t}-\widehat{y}_{t}\right|, \\
\mathrm{RMSE} & =\sqrt{\frac{1}{N} \sum_{t=1}^{N}\left(y_{t}-\widehat{y}_{t}\right)^{2},} \\
\mathrm{MAPE} & =\frac{1}{N} \sum_{t=1}^{N}\left|\frac{y_{t}-\widehat{y}_{t}}{y_{t}}\right| \times 100 \%,
\end{aligned}
$$




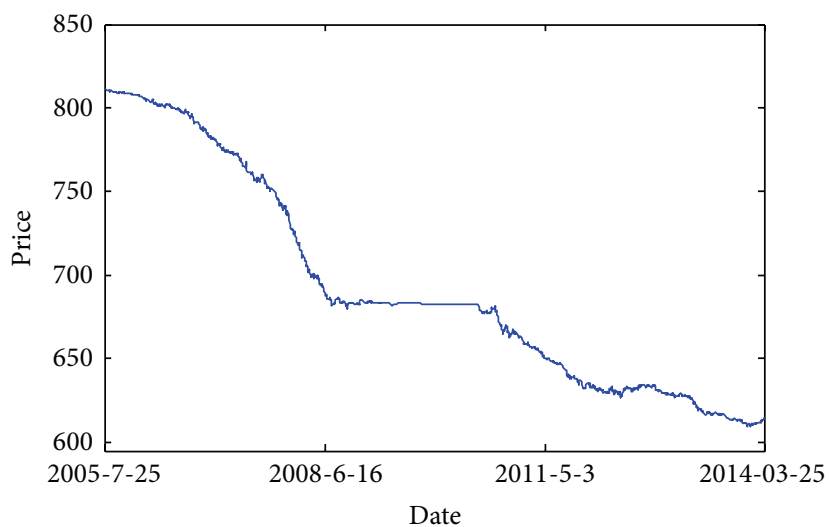

(a)

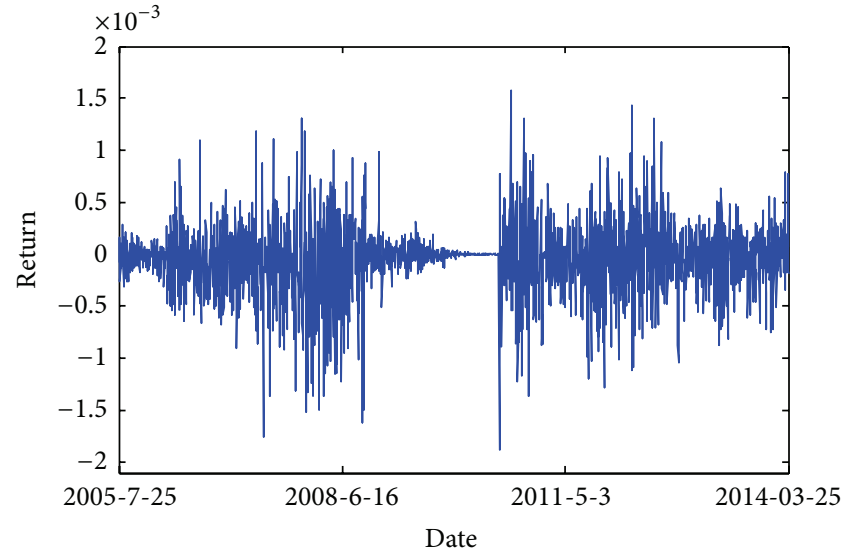

(b)

FIGURE 2: The RMB central parity rate against US dollar and its logarithmic return.

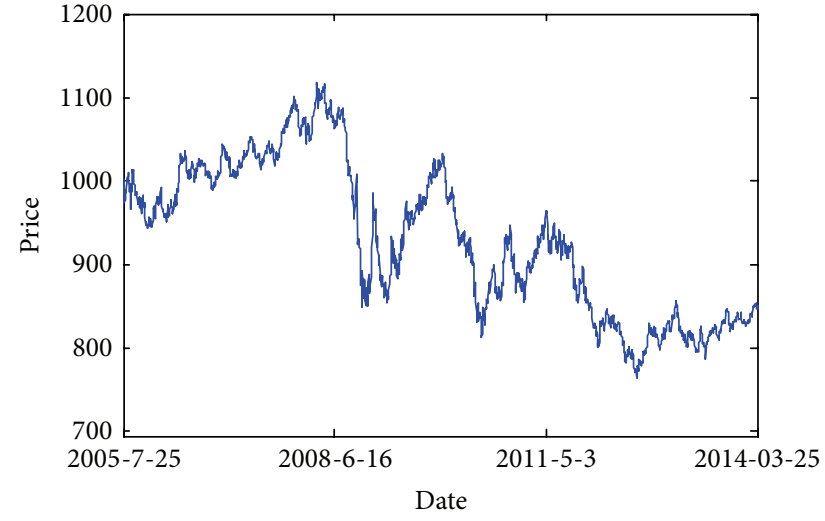

(a)

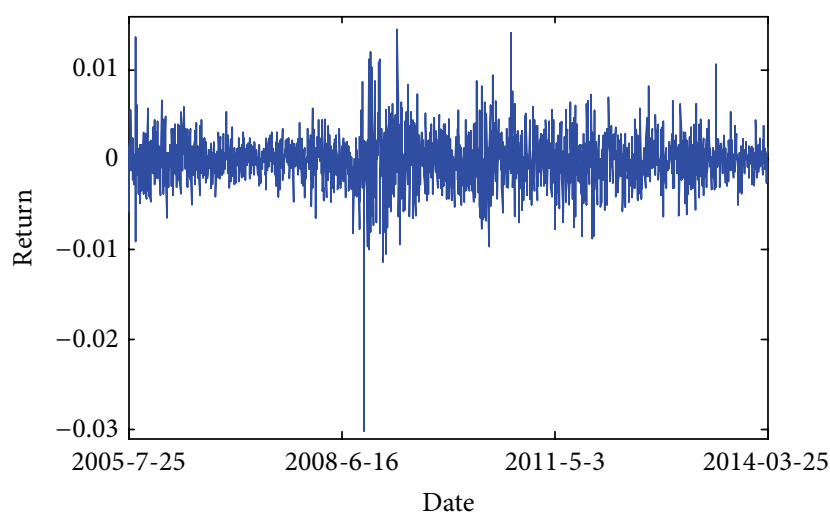

(b)

FIgURE 3: The RMB central parity rate against Euro and its logarithmic return.

where $y_{t}$ and $\hat{y}_{t}$ are the actual value and prediction value, respectively, and $N$ is the size of testing set.

MAE, RMSE, and MAPE are the measures of the deviation between actual values and prediction values. The values of these measures are desired to be as less as possible for an efficient forecasting performance.

\section{Data and Results}

4.1. Data Set. On July 21, 2005, China established a managed floating exchange rate regime based on market demand and supply with reference to a basket of currencies. To test the nonlinear combination model, this study selects the RMB central parity rate against US dollar (RMB/USD) and the RMB central parity rate against Euro (RMB/EUR). Besides, the sample interval is from July 25, 2005, to March 25, 2014. After excluding weekends and holidays, there remains a total of 2101 sample data. The sample data come from the State Administration of Foreign Exchange (http://www.safe.gov.cn/). The time series data are divided into training set, validation set, and testing set according to the ratio of $2001: 50: 50$.

In order to analyze the characteristics of the RMB exchange rate, we list the time series charts and logarithmic return diagrams of RMB/USD and RMB/EUR in Figures 2 and 3. The logarithmic return is defined as $R_{t}=\log P_{t}-$ $\log P_{t-1}$, where $P_{t}$ and $R_{t}$ stand for the price and return of the $\mathrm{RMB}$ exchange rate, respectively.

4.2. Prediction Results. It is well known that the RMB exchange rate has a stylized fact-the long memory feature (see, e.g., Wang and Xie [39]). Therefore, we first employ the detrended fluctuation analysis (DFA) method, which is proposed by Peng et al. [40] and is widely used to analyze financial markets $[39,41,42]$, to quantify the long memory of RMB exchange rate time series.

From Table 1, we find that the Hurst values of RMB/USD and RMB/EUR by the DFA method are 0.5914 and 0.5265 , respectively. That is to say, there is a weak long memory feature in RMB exchange rate against US dollar and $\mathrm{RMB}$ exchange rate against Euro. So, in this study, we use 


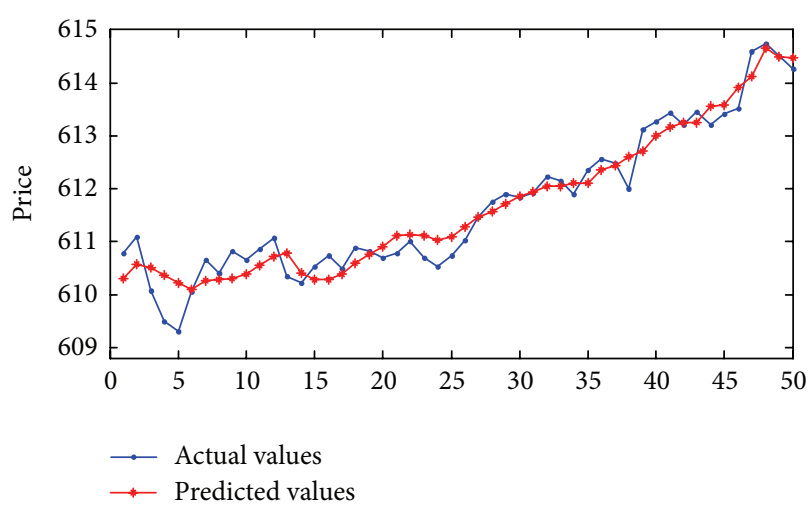

(a)

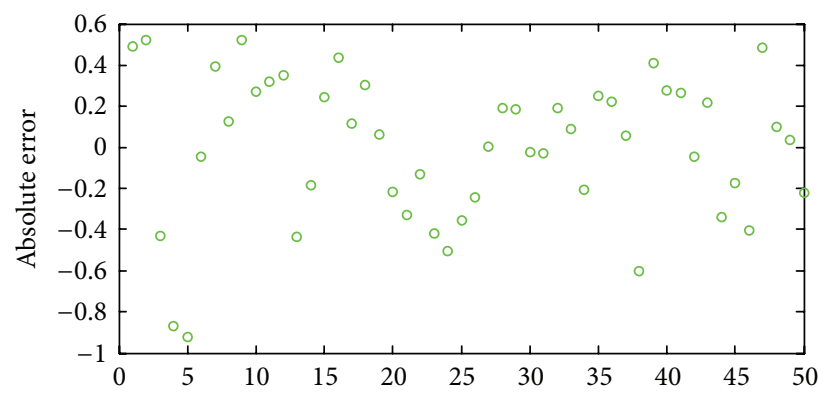

(b)

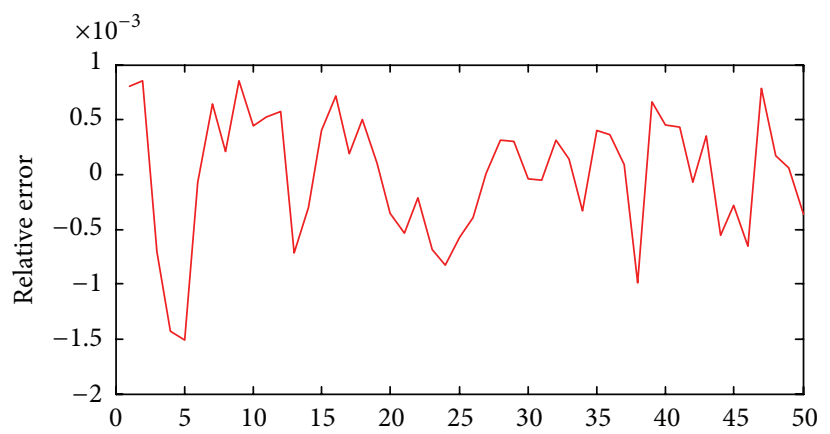

(c)

Figure 4: Comparison of the RMB/USD predicted values by the NCM-f model (ARFIMA, SVM, and BPNN) and the actual values and the corresponding errors. The absolute error is defined as the difference between the forecasting value and the actual value, and the relative error is defined as the ratio between the absolute error and the actual value.

TABLE 1: The Hurst values of returns for RMB/USD and RMB/EUR based on the DFA method.

\begin{tabular}{lcc}
\hline Series & RMB/USD & RMB/EUR \\
\hline Hurst & 0.5914 & 0.5265 \\
\hline
\end{tabular}

the ARFIMA model to predict the RMB exchange rate because it can actually capture the long memory of time series. In the ARFIMA model, we can calculate $d$ values according to (4), and they are 0.0914 and 0.0265 , respectively.

Because the building process of the prediction model of two kinds of the RMB exchange rates is the same, we only take the time series of RMB/USD as an example to build model and estimate parameters for testing the prediction of the nonlinear combination model. Considering that ARFIMA is suitable for stationary sequence and the fitting of nonstationary series makes the spurious regression phenomenon, we transform the nonstationary sequence to stationary. So, we use the EViews 6.0 software to perform the unit root test for testing the stability of the sequence and present the results for $\mathrm{RMB} / \mathrm{USD}$ in Table 2.

In Table 2, we can find that the ADF value of $P_{t}$ is -2.5742 , which is larger than the critical value under the significance level of $1 \%$; so, $P_{t}$ is a nonstationary series. In contrast, the $\mathrm{ADF}$ values of $R_{t}$ and $R_{f}$ are -43.9875 and
-35.3466, respectively, which are far smaller than the critical value under the significance level of $1 \%$; so, $R_{t}$ and $R_{f}$ are stationary series. Observing the autocorrelation coefficients and partial autocorrelation coefficients of the sequence, we estimate the parameters of the ARFIMA model according to the principle of minimizing AIC value. The ARFIMA model can be simulated by EViews 6.0 and $R$ softwares, respectively. We can obtain the forecast results of $y_{t}$ through the antilog transform of the $R_{f}$ sequence and denote $\widehat{y}_{t}^{(1)}$ by the result of the ARFIMA model.

Considering that a month has 20 trading days, we set the cycle of forecast analysis as 20 days. That is to say, we set the node number of input layer as 20 [43]. Since the network with one output layer is sufficient to model most of complex system with the specific accuracy [44] and we want to forecast the price on the next day, we set the node number of output layer as one. BPNN is sensitive to the number of neurons of hidden layer; so, the number of hidden layer nodes in the network has a great correlation with the prediction accuracy. It is well known that choosing the right number of neurons can improve the forecasting ability of BPNN greatly. In this paper, we use the trial and error method to determine the neuron number of hidden layer. Under the condition of the same parameters, we determine the optimal number of neurons in hidden layer based on the large number of repeated experiments with different number of hidden layer neurons. 


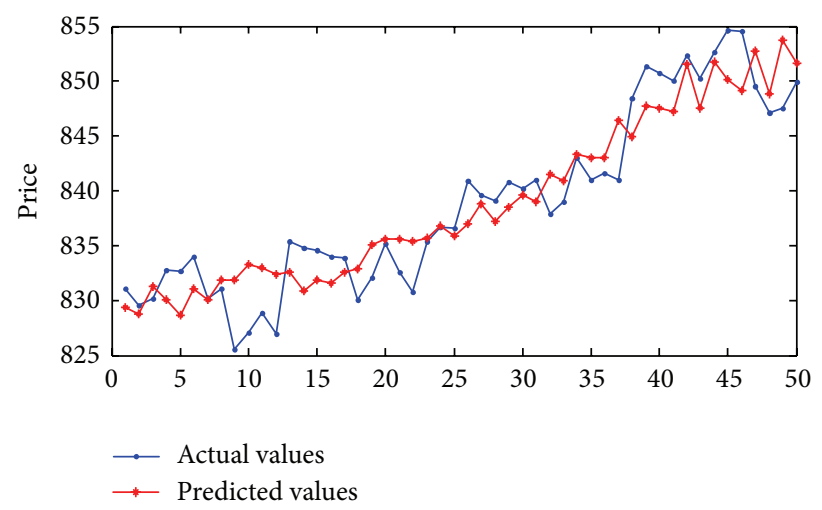

(a)

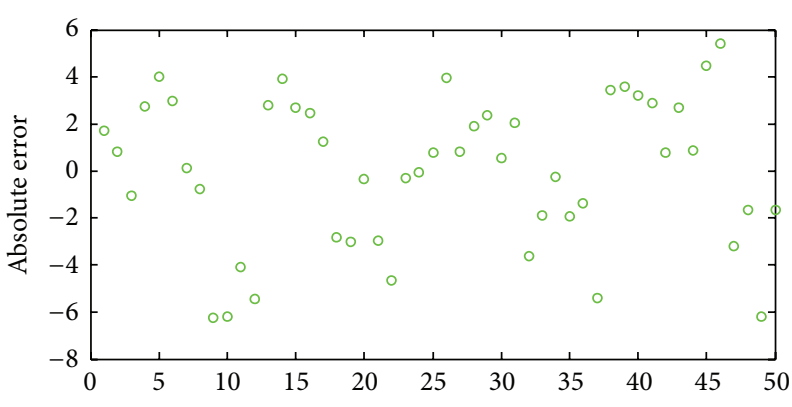

(b)

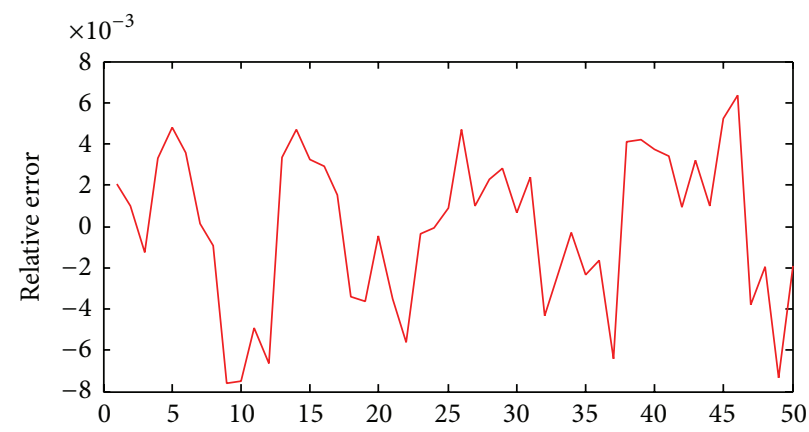

(c)

FIGURE 5: Comparison of the RMB/EUR predicted values by the NCM-f model (ARFIMA, SVM, and BPNN) and the actual values and the corresponding errors. The absolute error is defined as the difference between the forecasting value and the actual value, and the relative error is defined as the ratio between the absolute error and the actual value.

TABLE 2: Unit root test for RMB/USD.

\begin{tabular}{lcccccc}
\hline Series & ADF & $1 \%$ & $5 \%$ & $10 \%$ & $P$ value & Result \\
\hline$P_{t}$ & -2.5742 & -3.4333 & -2.8627 & -2.5674 & 0.0985 & Nonstationary \\
$R_{t}$ & -43.9875 & -3.4333 & -2.8627 & -2.5674 & 0.0001 & Stationary \\
$R_{f}$ & -35.3466 & -3.4333 & -2.8627 & -2.5674 & 0.0000 & Stationary \\
\hline
\end{tabular}

Notes: $P_{t}$ and $R_{t}$ stand for the prices and returns of RMB/USD, respectively, while $R_{f}$ denotes the $d$ th fractional difference series of the returns.

TABLE 3: Results of the trial and error method.

\begin{tabular}{lcccccccccc}
\hline Number & 1 & 2 & 3 & 4 & 5 & 6 & 7 & 8 & 9 & 10 \\
\hline Error & 3.4441 & 3.4246 & 2.5027 & 2.9670 & 2.9158 & 2.4029 & 2.6800 & 2.3356 & 2.4469 & 2.3974 \\
\hline Number & 11 & 12 & 13 & 14 & 15 & 16 & 17 & 18 & 19 & 20 \\
\hline Error & 3.5643 & 3.0448 & 2.9694 & 2.9802 & 2.7470 & 2.9302 & 3.3178 & 3.8917 & 2.9140 & 3.3352 \\
\hline
\end{tabular}

From Table 3, we can find that when the number of hidden layer neurons is eight, the mean square error of BPNN is the minimum (i.e., 2.3356). Therefore, we set the number of neurons in the hidden layer as eight.

The neural network toolbox and Libsvm toolbox of MATLAB software are utilized in the NCM model. The original data are scaled into the range of $[0,1.0]$ when we build the SVM model and BPNN model. Hereby, we denote $\widehat{y}_{t}^{(2)}$ by the result of the SVM model and denote $\widehat{y}_{t}^{(3)}$ by the result of the BPNN model.
After forecasting $\widehat{y}_{t}^{(1)}, \widehat{y}_{t}^{(2)}$, and $\widehat{y}_{t}^{(3)}$ by the models of ARFIMA, SVM, and BPNN, respectively, we combine the different prediction results by the nonlinear weighted mechanism. The comparisons between actual and predicted values for RMB/USD and RMB/EUR by the NCM model are, respectively, shown in Figures 4 and 5. In each figure, we also present the absolute error and the relative error, where the former is defined as the difference between the forecasting value and the actual value and the latter is defined as the ratio between the absolute error and the actual value. 
TABLE 4: The RMB/USD forecasting results using ARIMA, ARFIMA, SVM, BPNN, EWL, ARIMA-SVM, ARIMA-ANN, ARFIMA-SVM, ARFIMA-ANN, NCM-r, and NCM-f.

\begin{tabular}{lccc}
\hline & MAE & RMSE & MAPE (\%) \\
\hline ARIMA & 3.3793 & 4.1499 & 0.5516 \\
ARFIMA & 2.4994 & 3.1357 & 0.4079 \\
SVM & 2.4324 & 2.9654 & 0.3970 \\
BPNN & 2.2971 & 2.9268 & 0.3751 \\
EWL & 2.0106 & 2.8167 & 0.3280 \\
ARIMA-SVM & 2.2311 & 2.8101 & 0.3641 \\
ARIMA-ANN & 1.9873 & 2.6342 & 0.3243 \\
ARFIMA-SVM & 1.8724 & 2.5280 & 0.3055 \\
ARFIMA-ANN & 0.8442 & 1.0254 & 0.1379 \\
NCM-r & 0.3910 & 0.4987 & 0.0639 \\
NCM-f & 0.2849 & 0.3469 & 0.0466 \\
\hline
\end{tabular}

TABLE 5: The RMB/EUR forecasting results using ARIMA, ARFIMA, SVM, BPNN, EWL, ARIMA-SVM, ARIMA-ANN, ARFIMA-SVM, ARFIMA-ANN, NCM-r, and NCM-f.

\begin{tabular}{lccc}
\hline & MAE & RMSE & MAPE (\%) \\
\hline ARIMA & 9.7196 & 12.6395 & 1.1497 \\
ARFIMA & 9.1906 & 11.9829 & 1.0872 \\
SVM & 7.4141 & 9.6193 & 0.8772 \\
BPNN & 6.1349 & 7.8148 & 0.7263 \\
EWL & 7.1599 & 9.2979 & 0.8473 \\
ARIMA-SVM & 6.0254 & 7.3706 & 0.7171 \\
ARIMA-ANN & 5.5129 & 7.0651 & 0.6528 \\
ARFIMA-SVM & 5.4177 & 6.2094 & 0.6458 \\
ARFIMA-ANN & 4.8081 & 5.9508 & 0.5719 \\
NCM-r & 2.6946 & 3.2667 & 0.3210 \\
NCM-f & 2.6097 & 3.1159 & 0.3111 \\
\hline
\end{tabular}

From Figures 4 and 5, we can find that both the absolute error and the relative error fall into a small range, which indicates that the NCM model can capture both the linear and nonlinear features of the RMB exchange rate, and it can describe the RMB exchange rate time series accurately.

In order to compare the performances of different models, we apply ARIMA, ARFIMA, SVM, BPNN, EWL, ARIMA-SVM, ARIMA-ANN, ARFIMA-SVM, ARFIMAANN, NCM-r (ARIMA, SVM, and BPNN), and NCM-f (ARFIMA, SVM, and BPNN) to forecast the RMB/USD exchange rate and the RMB/EUR exchange rate. The prediction results obtained from the eleven models for the $\mathrm{RMB} / \mathrm{USD}$ are summarized in Table 4 , and the prediction results from the eleven models for the RMB/EUR are summarized in Table 5.

In order to evaluate the forecasting performances of the NCM model, we compare the NCM-f model with ARIMA, ARFIMA, SVM, BPNN, EWL, ARIMA-SVM, ARIMA-ANN, ARFIMA-SVM, ARFIMA-ANN, and NCM-r. Tables 4 and 5 present the performance results of MAE, RMSE, and MAPE for the eleven models. Obviously, the NCM-f model has much less errors than the other models, which implies that the NCM-f model has the best forecasting performance for the $\mathrm{RMB}$ exchange rate.

\section{Conclusion}

In real situations, the RMB exchange rate time series' characteristics are complex and unknown. Different models only can forecast time series from the aspects of linear or nonlinear characteristics, respectively. However, using single models cannot produce accurate forecasts for the RMB exchange rate. In this paper, a nonlinear combination model combining linear ARFIMA and nonlinear SVM and BPNN techniques was proposed and applied to the two RMB exchange rate time series (i.e., RMB/USD and RMB/EUR). The proposed nonlinear combination model not only can capture different forms of relationships in time series data more effectively but also can grasp the relationship between different models.

In order to demonstrate the effective prediction of nonlinear combination model, we use RMB/USD exchange rate and $\mathrm{RMB} / \mathrm{EUR}$ exchange rate as the empirical examples. We compare the NCM model with ARIMA, ARFIMA, SVM, BPNN, EWL, ARIMA-SVM, ARIMA-ANN, ARFIMASVM, and ARFIMA-ANN and find that the NCM model outperforms all other models. This study indicates that we develop an effective nonlinear combination model to generate more accurate forecasts for an extremely complicated exchange rate time series by combining different models. In addition, the NCM- $f$ model is more efficient than the NCM$r$ model. The reason is that RMB/USD exchange rate and $\mathrm{RMB} / \mathrm{EUR}$ exchange rate have a weak long memory feature, and the ARFIMA model can predict more accurately than the ARIMA model. It is worth noting that the nonlinear combination forecasting model is an indeed powerful risk prediction tool for managers and investors. The implication of the wide applications of nonlinear combination model is that the forecasting quality can be improved in many areas where data sets have the complex relationships.

\section{Conflict of Interests}

The authors declare that there is no conflict of interests regarding the publication of this paper.

\section{Acknowledgments}

The authors are grateful to the editor and three reviewers for their valuable suggestions that significantly improved the quality of the paper. The research is supported by the National Natural Science Foundation of China under Grant nos. 71373072 and 71340014, the Specialized Research Fund for the Doctoral Program of Higher Education under Grant no. 20130161110031, the Hunan Provincial Innovation Foundation for Postgraduate under Grant no. CX2013A006, and the Foundation for Innovative Research Groups of the National Natural Science Foundation of China under Grant no. 71221001 . 


\section{References}

[1] Y. Ding and Y. S. Ying, "The dynamic analysis of exchange rate risk and China's export trade," Journal of Finance and Economics, vol. 26, no. 4, pp. 91-98, 2011 (Chinese).

[2] A. Grossmann and M. W. Simpson, "Forecasting the Yen/U.S. dollar exchange rate: empirical evidence from a capital enhanced relative PPP-based model," Journal of Asian Economics, vol. 21, no. 5, pp. 476-484, 2010.

[3] Z. H. Hu, "The practical equilibrium RMB exchange rate: decisive factors and cooperative examination," Journal of Asian Economics, vol. 20, no. 5, pp. 54-59, 2005.

[4] X. F. Dai and Q. X. Xiao, "Time series analysis applied in prediction of RMB's exchange rate," Journal of University of Shanghai for Science and Technology, vol. 27, no. 4, pp. 341-344, 2005 (Chinese).

[5] S. Q. Xu and Y. M. Li, "The RMB exchange rate forecasting about Basket of currencies-based on empirical methods of ARMA model," World Economic Papers, vol. 3, pp. 30-40, 2007 (Chinese).

[6] L. Ma and Q. H. Xu, "Research on trend predication of foreign exchange and its application to foreign exchange financing business of commercial banks based on model ARMA," Journal of Southwest China Normal University (Natural Science Edition), vol. 34, no. 2, pp. 114-118, 2009 (Chinese).

[7] J. G. Sun and H. H. Wu, "Nonlinear analysis of the foreign exchange market based on the R/S method," Statistics and Decision, no. 16, pp. 70-72, 2006 (Chinese).

[8] R. X. Rong, "Research on nonlinear characteristics of RMB exchange rate-based on the empirical test of R/S analysis method," Journal of Shanxi Finance and Economics University, vol. 30, no. 10, pp. 107-111, 2008 (Chinese).

[9] C. Xie, S. Long, M. H. Liu, and B. Sun, "An empirical analysis of the nonlinear correlation structure of RMB exchange rate," Statistics and Decision, vol. 8, pp. 107-110, 2010 (Chinese).

[10] X. C. Yang and Y. R. Wu, "The nonlinear forecasting analysis of exchange rate based on support vector machine (SVM)," Statistics and Decision, no. 18, pp. 13-16, 2010 (Chinese).

[11] C. Kadilar, M. Simsek, and C. H. Aladag, "Forecasting the exchange rate series with ANN: the case of Turkey," Istanbul University Journal of Econometrics and Statistics, vol. 9, no. 1, pp. 17-29, 2009.

[12] C. H. Aladag, E. Egrioglu, C. Bal, and B. Alptekin, "Forecasting TL/Chinese RMB and TL/USD exchange rates with feed forward neural networks," in Proceeding of the 8th International Symposium of Statistics Abstracts, pp. 26-27, Eskisehir, Turkey, October 2012.

[13] C. H. Aladag and M. M. Marinescu, "Tl/Euro and Leu/Euro exchange rates forecasting with artificial neural networks," Journal of Social and Economic Statistics, vol. 2, no. 2, pp. 1-6, 2013.

[14] Y. Kajitani, K. W. Hipel, and A. I. McLeod, "Forecasting nonlinear time series with feed-forward neural networks: a case study of Canadian lynx data," Journal of Forecasting, vol. 24, no. 2, pp. 105-117, 2005.

[15] C. Panda and V. Narasimhan, "Forecasting exchange rate better with artificial neural network," Journal of Policy Modeling, vol. 29, no. 2, pp. 227-236, 2007.

[16] G. P. Zhang, "Time series forecasting using a hybrid ARIMA and neural network model," Neurocomputing, vol. 50, pp. 159175, 2003.
[17] P. F. Pai and C. S. Lin, "A hybrid ARIMA and support vector machines model in stock price forecasting," Omega, vol. 33, no. 6, pp. 497-505, 2005.

[18] F. Alpaslan, O. Cagcag, C. H. Aladag, U. Yolcu, and E. Egrioglu, "A novel seasonal fuzzy time series method," Hacettepe Journal of Mathematics and Statistics, vol. 4, no. 3, pp. 375-385, 2012.

[19] F. Alpaslan and O. Cagcag, "A seasonal fuzzy time series forecasting method based on Gustafson-Kessel fuzzy clustering," Journal of Social and Economic Statistics, vol. 2, no. 1, pp. 1-13, 2012.

[20] O. Cagcag Yolcu, "A hybrid fuzzy time series approach based on fuzzy clustering and artificial neural network with single multiplicative neuron model," Mathematical Problems in Engineering, vol. 2013, Article ID 560472, 9 pages, 2013.

[21] I. Luna, S. Soares, and R. Ballini, "A constructive-fuzzy system modeling for time series forecasting," in Proceedings of the International Joint Conference on Neural Networks (IJCNN '07), pp. 2908-2913, Orlando, Fla, USA, August 2007.

[22] I. Rojas, O. Valenzuela, F. Rojas et al., "Soft-computing techniques and ARMA model for time series prediction," Neurocomputing, vol. 71, no. 4-6, pp. 519-537, 2008.

[23] W. K. Wong, M. Xia, and W. C. Chu, "Adaptive neural network model for time-series forecasting," European Journal of Operational Research, vol. 207, no. 2, pp. 807-816, 2010.

[24] L. Zhao and Y. Yang, "PSO-based single multiplicative neuron model for time series prediction," Expert Systems with Applications, vol. 36, no. 2, pp. 2805-2812, 2009.

[25] C. H. Aladag, E. Egrioglu, and C. Kadilar, "Forecasting nonlinear time series with a hybrid methodology," Applied Mathematics Letters, vol. 22, no. 9, pp. 1467-1470, 2009.

[26] E. Egrioglu, C. H. Aladag, and C. Kadilar, New Developments in Artificial Neural Networks Research, Nova Publisher, New York, NY, USA, 2011.

[27] C. H. Aladag, E. Egrioglu, and C. Kadilar, "Improvement in forecasting accuracy using the hybrid model of ARFIMA and feed forward neural network," American Journal of Intelligent Systems, vol. 2, no. 2, pp. 12-17, 2012.

[28] O. Valenzuela, I. Rojas, F. Rojas et al., "Hybridization of intelligent techniques and ARIMA models for time series prediction," Fuzzy Sets and Systems, vol. 159, no. 7, pp. 821-845, 2008.

[29] M. Khashei and M. Bijari, "A novel hybridization of artificial neural networks and ARIMA models for time series forecasting," Applied Soft Computing Journal, vol. 11, no. 2, pp. 26642675, 2011.

[30] C. W. J. Granger and R. Joyeux, "An introduction to longmemory time series models and fractional differencing," Journal of Time Series Analysis, vol. 1, no. 1, pp. 15-29, 1980.

[31] J. R. M. Hosking, "Fractional differencing," Biometrika, vol. 68, no. 1, pp. 165-176, 1981.

[32] C. Ellis, "Estimation of the ARFIMA (p, d, q) fractional differencing parameter (d) using the classical rescaled adjusted range technique," International Review of Financial Analysis, vol. 8, no. 1, pp. 53-56, 1999.

[33] G. E. P. Box and G. M. Jenkins, Time Series Analysis, Forecasting and Control, Holden-Day, San Francisco, Calif, USA, 1976.

[34] V. N. Vapnik, The nature of statistical learning theory, Springer, Berlin, Germany, 2nd edition, 2000.

[35] D. E. Rumelhart and J. L. McClelland, Parallel Distributed Proeessing: Explorations in the Microstructure of Cognition, MIT, Cambridge, Mass, USA, 1986. 
[36] M. Hibon and T. Evgeniou, "To combine or not to combine: selecting among forecasts and their combinations," International Journal of Forecasting, vol. 21, no. 1, pp. 15-24, 2005.

[37] M. R. Hassan, B. Nath, and M. Kirley, "A fusion model of HMM, ANN and GA for stock market forecasting," Expert Systems with Applications, vol. 33, no. 1, pp. 171-180, 2007.

[38] C. H. Aladag, E. Egrioglu, and U. Yolcu, "Forecast combination by using artificial neural networks," Neural Processing Letters, vol. 32, no. 3, pp. 269-276, 2010.

[39] G. J. Wang and C. Xie, "Cross-correlations between Renminbi and four major currencies in the Renminbi currency basket," Physica A Statistical Mechanics and Its Applications, vol. 392, no. 6, pp. 1418-1428, 2013.

[40] C.-K. Peng, S. V. Buldyrev, S. Havlin, M. Simons, H. E. Stanley, and A. L. Goldberger, "Mosaic organization of DNA nucleotides," Physical Review E, vol. 49, no. 2, pp. 1685-1689, 1994.

[41] G. J. Wang, C. Xie, P. Zhang, F. Han, and S. Chen, "Dynamics of foreign exchange networks: a time-varying copula approach," Discrete Dynamics in Nature and Society, vol. 2014, Article ID 170921, 11 pages, 2014.

[42] G. J. Wang and C. Xie, "Cross-correlations between WTI crude oil market and U.S. stock market: a perspective from econophysics," Acta Physica Polonica B, vol. 43, no. 10, pp. 20212036, 2012.

[43] X. L. Zhu and P. Li, "Research on fitting and forecasting the RMB exchange rate by BP neural net," Journal of South-Central University for Nationalities (Natural Science Edition), vol. 29, no. 3, pp. 113-120, 2010 (Chinese).

[44] Y. Chauvin and D. E. Rumelhart, Backpropagation: Theory, Architectures, and Applications, Psychology Press, London, UK, 1995. 


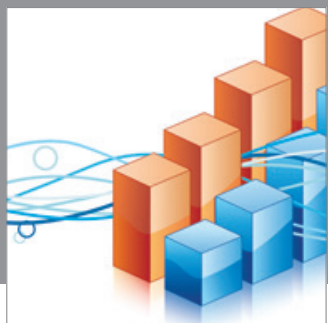

Advances in

Operations Research

mansans

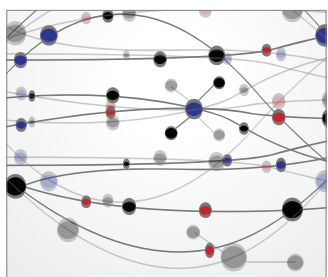

The Scientific World Journal
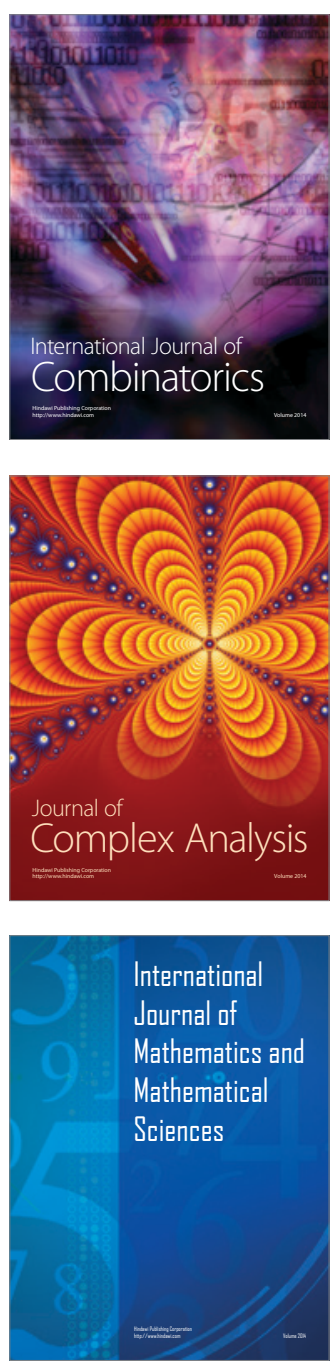
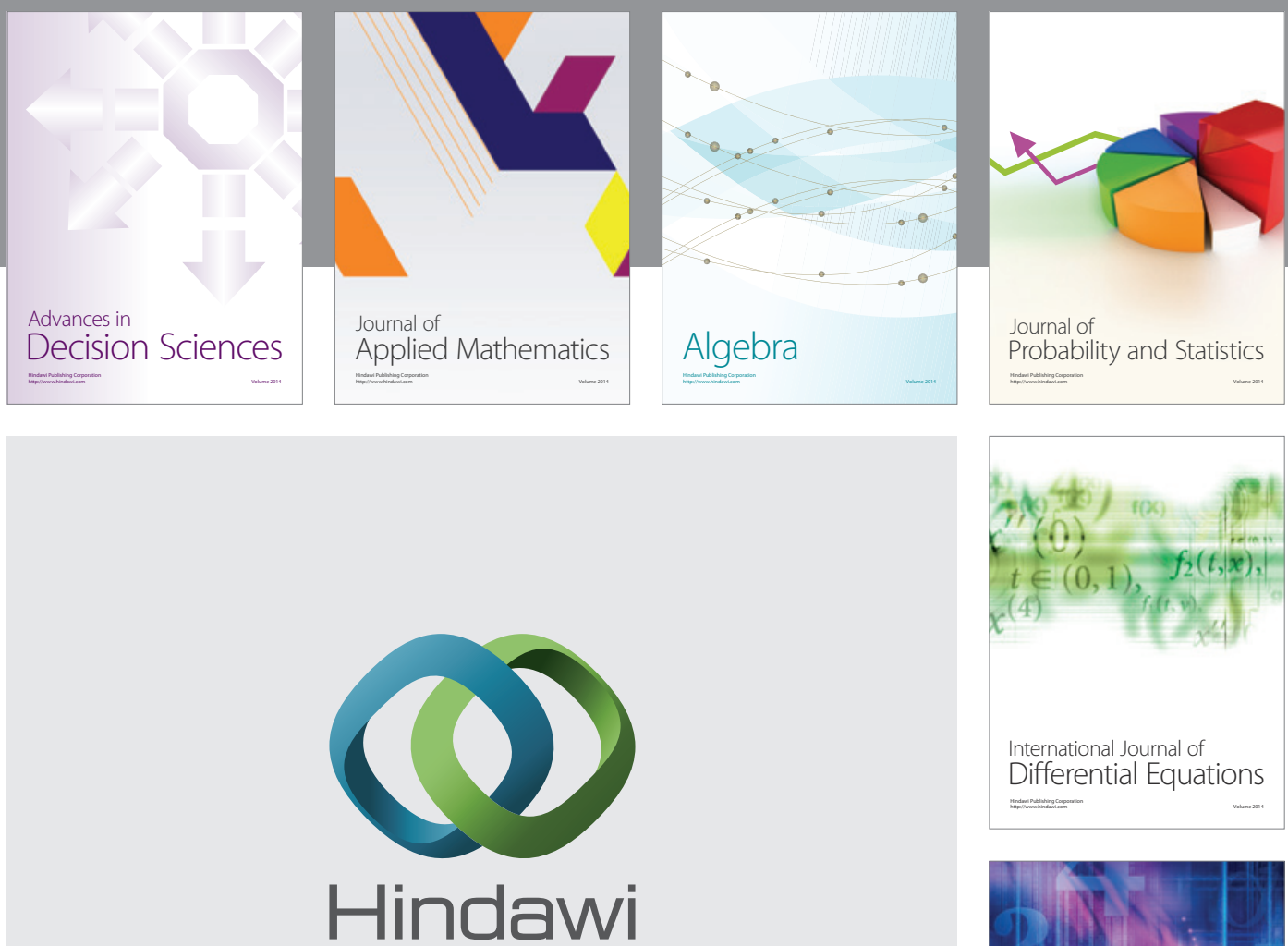

Submit your manuscripts at http://www.hindawi.com
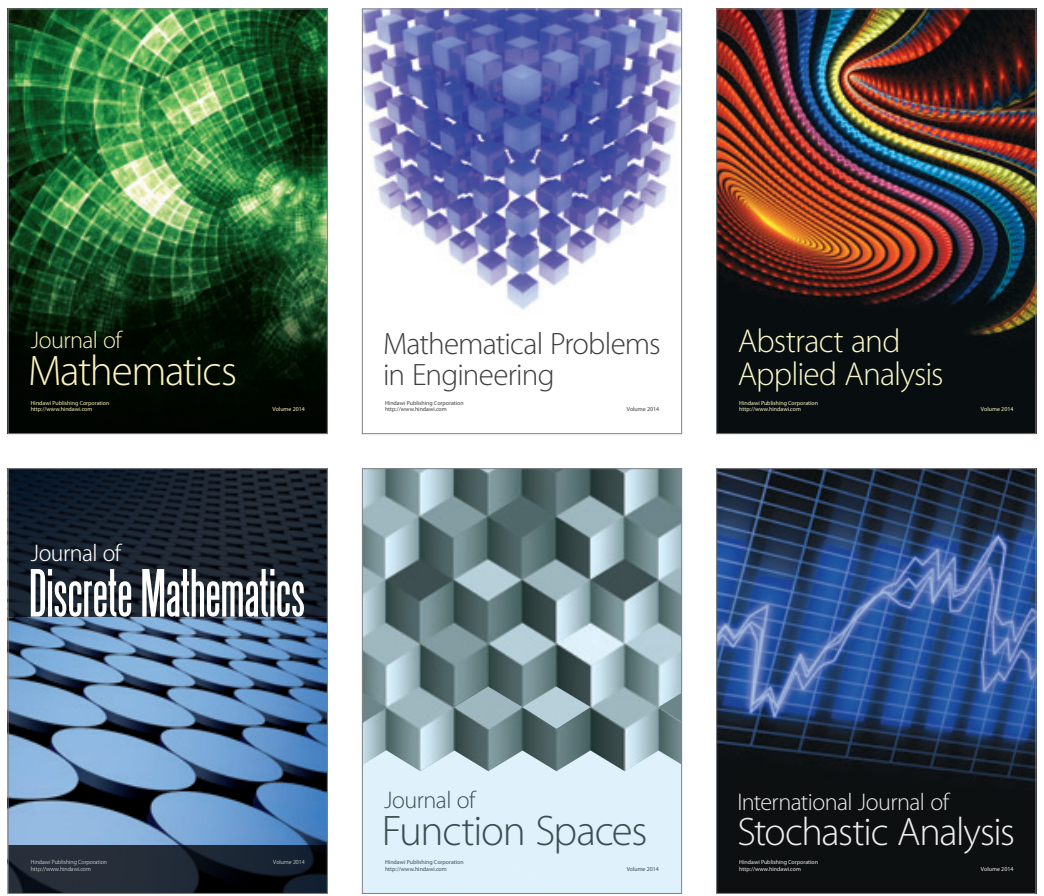

Journal of

Function Spaces

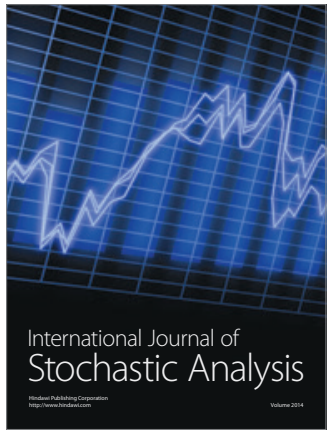

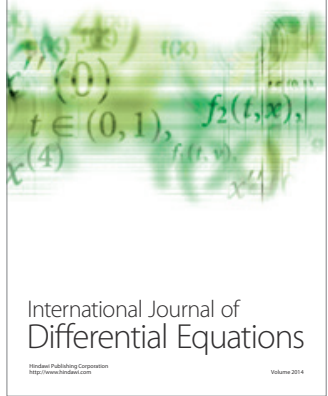
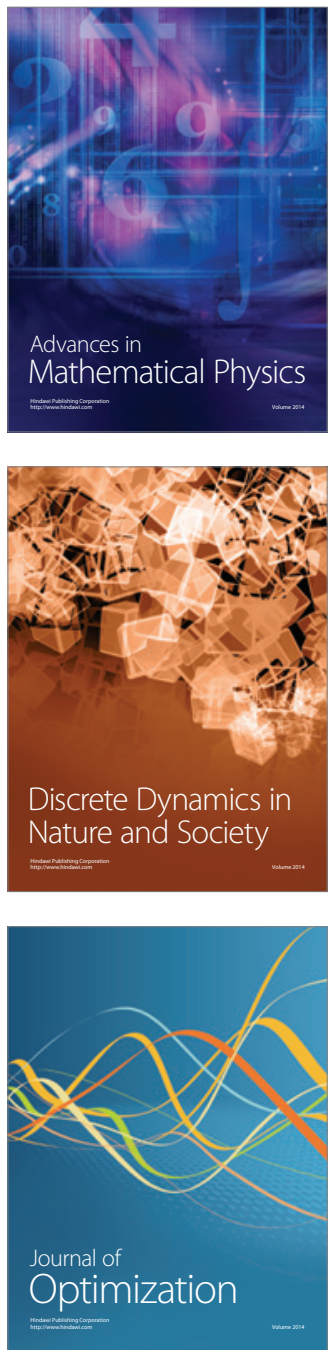